ECOLE PRATIQUE DES HAUTES ÉTUDES - SORBONNE SIXIEMME SECTION : SCIENCES ECONOMIQUES ET SOCIALES

\title{
CONGRÈS \\ ET \\ COLLOQUES
}

I

PA R I S MOUTON \& CO LA HAYE M C M L X 


\section{FIRST INTERNATIONAL CONFERENCE OF ECONOMIC HISTORY}

\section{CONTRIBUTIONS}

A. Industrialisation as a factor in economic growth after 1700

B. Comparative study of large-scale agricultural enterprise in post-medieval times

\section{COMMUNICATIONS}




\section{PREMIÈRE CONFÉRENCE INTERNATIONALE D'HISTOIRE ECONOMIQUE}

\section{CONTRIBUTIONS}

A. L'industrialisation comme facteur de la croissance économique depuis 1700

B. Étude comparée du grand domaine depuis la fin du Moyen Age

\section{COMMUNICATIONS}


Bureau:

F. BRAUDEL, Paris.

M. M. POSTAN, Cambridge.

E. SÖDERLUND, Stockholm.

\section{Cet ouvrage a été publié}

avec le généreux concours

de l'UNESCO

(C) I960, Mouton \& Co, Publishers, Paris-The Hague. 